\title{
Rheumatoid arthritis patients on persistent moderate disease activity on biologics have adverse 5-year outcome compared to persistent low-remission status and represent a heterogeneous group
}

\author{
Irini Genitsaridi ${ }^{1}$, Irini Flouri ${ }^{1}$, Dimitris Plexousakis ${ }^{2}$, Konstantinos Marias ${ }^{3}$, Kyriaki Boki ${ }^{4}$, Fotini Skopouli ${ }^{5}$,
} Alexandros Drosos ${ }^{6}$, George Bertsias ${ }^{1}$, Dimitrios Boumpas ${ }^{7}$ and Prodromos Sidiropoulos ${ }^{1 *}$ (i)

\begin{abstract}
Background: The long-term outcome of rheumatoid arthritis (RA) patients who in clinical practice exhibit persistent moderate disease activity (pMDA) despite treatment with biologics has not been adequately studied. Herein, we analyzed the 5-year outcome of the PMDA group and assessed for within-group heterogeneity.

Methods: We included longitudinally monitored RA patients from the Hellenic Registry of Biologic Therapies with persistent (cumulative time $\geq 50 \%$ of a 5 -year period) moderate (pMDA, $3.2<$ DAS28 $\leq 5.1$ ) or remission/low (pRLDA, DAS28 $\leq 3.2$ ) disease activity. The former was further classified into persistent lower-moderate (pIMDA, DAS28 < 4.2) and higher-moderate (phMDA, DAS28 $\geq 4$.2) subgroups. Five-year trajectories of functionality (HAQ) were the primary outcome in comparing pRLDA versus PMDA and assessing heterogeneity within the pMDA subgroups through multivariable mixed-effect regression. We further compared serious adverse events (SAEs) occurrence between the two groups.

Results: We identified 295 patients with pMDA and 90 patients with pRLDA, the former group comprising of pIMDA $(n=133,45 \%)$ and phMDA $(n=162,55 \%)$. pMDA was associated with worse 5 -year functionality trajectory than pRLDA (+ $0.27 \mathrm{HAQ}$ units, Cl 95\% + 0.22 to $+0.33 ; p<0.0001$ ), while the phMDA subgroup had worse 5 -year functionality than pIMDA (+ $0.26 \mathrm{HAQ}$ units, $\mathrm{Cl} 95 \% 0.18$ to $0.36 ; p<0.0001$ ). Importantly, higher persistent disease activity was associated with more SAEs [pRLDA: $0.2 \pm 0.48$ vs pMDA: $0.5 \pm 0.96, p=0.006$; pIMDA: $0.32 \pm 0.6$ vs phMDA: $0.64 \pm 1.16, p=0.038$ ]. Male gender $(p=0.017)$, lower baseline DAS28 $(p<0.001)$, HAQ improvement $>0.22$ $(p=0.029)$, and lower average DAS28 during the first trimester since treatment initiation $(p=0.001)$ independently predicted grouping into pRLDA.

(Continued on next page)
\end{abstract}

\footnotetext{
* Correspondence: sidiropp@uoc.gr

${ }^{1}$ Rheumatology and Clinical Immunology Department, School of Medicine at University of Crete, Voutes, 71110 Heraklion, Greece

Full list of author information is available at the end of the article
}

C C The Author(s). 2020 Open Access This article is licensed under a Creative Commons Attribution 4.0 International License, which permits use, sharing, adaptation, distribution and reproduction in any medium or format, as long as you give appropriate credit to the original author(s) and the source, provide a link to the Creative Commons licence, and indicate if changes were made. The images or other third party material in this article are included in the article's Creative Commons licence, unless indicated otherwise in a credit line to the material. If material is not included in the article's Creative Commons licence and your intended use is not permitted by statutory regulation or exceeds the permitted use, you will need to obtain permission directly from the copyright holder. To view a copy of this licence, visit http://creativecommons.org/licenses/by/4.0/ The Creative Commons Public Domain Dedication waiver (http://creativecommons.org/publicdomain/zero/1.0/) applies to the data made available in this article, unless otherwise stated in a credit line to the data. 
(Continued from previous page)

Conclusions: In clinical practice, RA patients with pMDA while on bDMARDs have adverse long-term outcomes compared to lower disease activity status, while heterogeneity exists within the pMDA group in terms of 5-year functionality and SAEs. Targeted studies to better characterize pMDA subgroups are needed, in order to assist clinicians in tailoring treatments.

Keywords: Persistent moderate rheumatoid arthritis, Biologics, Functionality, Serious adverse events, Heterogeneity

\section{Background}

Rheumatoid arthritis (RA) disease activity state is associated with long-term prognosis [1]. According to the widely accepted treat-to-target (T2T) strategy, the aim of treatment in RA is to improve patients' health-related quality of life by abrogation of inflammatory burden [2]. T2T approach in RA aims remission or low disease activity, evaluated by composite indexes such as the disease activity score of 28 joint counts (DAS28) [3]. Biologic disease-modifying antirheumatic drugs (bDMARDs) are potent agents which control inflammation and improve prognosis. Their clinical effectiveness has been extensively reported in controlled clinical trials and registries. Data from registries have shown that $15-35 \%$ of patients treated with TNFa inhibitors (TNFis) achieve remission at 12 months [4-7] and figures are comparable with non-TNFis such as tocilizumab [8]. Interestingly, approximately 30-50\% of patients although improve disease activity, they still have moderate disease activity (MDA) after treatment with the first bDMARD [6].

Outcomes of patients with residual MDA while on treatment are available only from trials focusing on early-onset disease treated with conventional synthetic DMARDs (csDMARDs) $[9,10]$ and a pooled analysis of randomized controlled clinical trials of TNF is reported on early (1-year) outcomes [1]. Moreover, most of the registries report on disease activity status at prespecified time-points after treatment, while data indicative of long-term longitudinal disease activity course (i.e., timeaveraged disease activity) are limited. To this end, data for RA patients on bDMARDs having persistently MDA are scant. Rheumatologists in clinical practice often confront with patients who have residual disease activity even after switching biologic disease-modifying drugs. Therefore, and given the lack of data for this group, we sought to assess the long-term outcome of RA patients on bDMARDs who exhibit persistent moderate disease activity (pMDA) in clinical practice context.

Herein, we analyzed data from the Hellenic Registry of Biologic Therapies to evaluate the long-term ( 5 years) outcome of RA patients on bDMARDs who exhibit pMDA. We aimed to compare the functional status (HAQ) at 5 years and its longitudinal course (trajectories) in patients with pMDA versus those on persistent lower inflammatory burden (pRLDA). Additionally, we compared serious adverse events (SAEs) occurrence during the course of the follow-up between the two groups. We finally assessed for clinical heterogeneity within pMDA and looked for early predictors of patients' classification into the distinct persistent disease activity groups.

\section{Methods}

Data source

The Hellenic Registry of Biologic Therapies (HeRBT) was established in 2004 as a multicenter ( 7 centers) prospective observational cohort of patients with inflammatory arthritides [6]. RA and spondyloarthritis patients are included in the HeRBT at the initiation of first bDMARD and are followed-up for as long as they receive bDMARDs. Detailed analysis of protocol is given elsewhere $[6,11]$. Treatment decisions (bDMARD selection, co-medication, dosage adjustments/switches) were made at the discretion of treating rheumatologists based on clinical assessments, national guidelines, and patient's preferences. All adverse events were reported using a separate form in every visit by patients and treating physicians and details regarding their seriousness, healthcare utilization, and outcome were recorded. Ethical approval was obtained from the Institutional Review-Board of the University Hospital of Heraklion, Crete (decision number 1476/20-03-2012), along with participants' informed consents.

\section{Patients}

We analyzed all adult patients diagnosed with RA who were registered for the first time in HeRBT until 31 May 2013 and had continuous follow-up for at least 5 years, irrespective of bDMARD treatment switches. Data were censored when patients completed 5 years of follow-up.

\section{Longitudinal clinical data}

Data harmonization was performed to summarize longitudinal disease activity (assessed by DAS28 index) and functionality (assessed by Health Assessment Questionnaire (HAQ)) assessments in clinically relevant therapy time (TT) intervals. Specifically, we defined a total of eight TT intervals that spanned sequentially over the 5 years follow-up period, every 6 months for the first 2 
years and every year afterwards. DAS28 and HAQ averages were computed for each patient, in each TT interval, from all clinical assessments conducted in the specific time period. In addition, the patients' serious adverse events (SAEs) were computed during the 5 years of follow-up. SAEs were defined as adverse events that required hospitalization and/or intravenous antibiotics, resulted in significant disability, and/or were lifethreatening or fatal.

\section{Definitions and clustering by persistent disease activity}

Patients were classified into persistent low (pRLDA) and persistent moderate (pMDA) disease activity groups, if they correspondingly had DAS28 $\leq 3.2$ and $3.2<$ DAS2 $8 \leq 5.1$ for cumulative time percentage $\geq 50 \%$ of the 5-year follow-up time. Cumulative time percentage (CTP) of a DAS28 range was defined as the ratio of TT intervals that have DAS28 in the specific range. A patient was clustered in a persistent disease activity group when a specific DAS28-range occurred for CTP $\geq 50 \%$ or equivalently for at least 4 out of the 8 TT intervals (any of them).

To assess for within-group heterogeneity, patients with pMDA were further sub-divided into persistent lowermoderate (plMDA) and higher-moderate (phMDA) disease activity groups, when they fulfilled the additional criterion of having persistent DAS28 $<4.2$ and DAS28 $\geq$ 4.2 , respectively, for CTP $\geq 50 \%$, as defined above. Any conflicting cases, where patients could be classified in two different persistent disease activity groups, were resolved by the preference policy of the worst-case scenario (classification in the higher disease activity group).

\section{Statistical analysis}

Summary descriptive measures were used on baseline characteristics of the persistent disease activity groups. Non-parametric hypothesis tests (Kruskal-Wallis, Wilcoxon rank-sum, and chi-squared, as appropriate) were used to compare groups' differences at baseline characteristics (Bonferroni-corrected $p$ value $=0.0019$ to account for multiple comparisons) and also to compare groups' 5-year outcomes regarding functional status (HAQ) and cumulative serious adverse events (SAEs). To estimate the required sample size to compare functionality, we formulated the null hypothesis that functionality (HAQ) at 5 years would not differ significantly between pRLDA and pMDA groups (i.e., difference in HAQ $\leq 0.22$ units). To reject this null hypothesis with $80 \%$ power $(\alpha=0.05)$ and assuming 0.5 mean HAQ $(0.5$ std) in the pMDA and 0.28 in the pRLDA group and patient allocation ratio pRLDA to pMDA to be $1: 2$, the estimated required sample size was at least 61 and 122 patients in the pRLDA and PMDA groups, respectively.
Multivariable mixed-effect regression analysis was used to associate the persistent disease activity groups pRLDA and pMDA with different 5-year functionality trajectories. Additionally, multivariable mixed-effect regression was performed for pMDA group to assess for clinical heterogeneity (5-year functionality trajectories) between plMDA and phMDA subgroups. In the analyses, HAQ was modeled as the dependent outcome variable while the persistent disease activity group (or subgroup) was modeled as fixed-effect variable (categorical, dummy-coded with reference-category the lower persistent disease activity group). We accounted for individual patient variability with a random effect on the patient level (random intercept for each patient) and adjusted for possible confounding effects of gender, age, and disease duration (fixed-effects). Time in treatment course was modeled as fixed-effect categorical variable (dummy-coded, 9-values, reference-category baseline, remaining values represent the 8 TT intervals). This time representation was selected to model the change in HAQ in each TT interval compared to baseline. Alternative representations of groups' interactions with time did not yield significantly better results on comparison metrics of AIC, BIC, and negative log-likelihood.

Multivariable logistic regression was also performed to analyze for early predictors that classify in pMDA (versus pRLDA) group. The model was adjusted for gender, age (per year), disease duration (per year), and previous csDMARDs at baseline (binary, true for csDMARDs < 2 ). The model also included the following patient's characteristics from baseline and first therapy semester: baseline disease activity (DAS28), baseline functionality (HAQ), anti-TNF treatment initiation, average disease activity in first semester (DAS28-average), and functionality difference $(\triangle \mathrm{HAQ})$ in first semester's average (HAQ-average) from baseline (binary, true for difference $<-0.22$ ). Predictive efficiency of the model was evaluated using the 10 -fold cross-validation process (90\% training set, $10 \%$ test set, and 10 repetitions without resubstitution) in the samples of the analysis cohort, in order to avoid overfitting and selection bias. The efficiency metrics, accuracy (ACC), sensitivity or true positive rate (TPR), specificity or true negative rate (TNR), and the area under the receiver-operating-characteristic curve (AUC), were averaged from 10 independent datasets extracted from the cross-validation process.

\section{Missing data management}

Patients who had proportionally more missing than existing DAS28-assessments were excluded in order to maintain data quality. Missing DAS28-data in the rest of the patients were imputed with a multivariable mixedeffect regression model, due to the time-dependent nature of the longitudinal DAS28-data (repeated correlated 
DAS28-measurements in the same patients). The model was adjusted on gender, age, and disease duration (fixedeffects) and included a random effect on the patient level and a fixed-effect variable for time (as in the aforementioned mixed-effect model). All analyses were performed in MATLAB 9.2 statistical toolbox.

\section{Results}

\section{Cohort characteristics}

The analysis cohort included 385 patients from the HeRBT registry that fulfilled two criteria, (a) they had at least 5 years follow-up and (b) they exhibited persistent low or moderate disease. This cohort was selected out of the 1466 RA patients included in the registry until May 2013, after excluding 763 patients due to $<5$ years follow-up, 166 patients having missing longitudinal DAS28-data >50\%, 142 exhibiting persistent high inflammatory burden, and 10 patients not exhibiting any persistent disease activity level. This was a cohort mostly with established RA (mean disease duration 9.2 years), 70 patients had disease duration $<2$ years, and the mean monitoring duration was 7.5 years (Table 1). Patients were treated with an average of $2.34( \pm 1.13)$ csDMARDs prior to first bDMARD, while 279 (72\%) were on combination with methotrexate. At the end of 5 years, 208 (54\%) patients received a second and $99(26 \%)$ received a third sequential bDMARD.

\section{Persistent disease activity groups characteristics}

A total 90 (23\%) and 295 (77\%) patients were categorized in the pRLDA and pMDA groups, respectively. Patients in the pMDA group were further categorized in subgroups plMDA and phMDA including 133 (45\%) and $162(55 \%)$ patients, respectively (Table 1$)$. Patients in the pMDA group were older ( $58 \pm 12$ vs $52 \pm 13$ years; $p<$ 0.0019 Bonferroni-corrected) and more frequently females $(82 \%$ vs $58 \%, p<0.0019)$ as compared to pRLDA. At inclusion, patient disease activity (mean DAS28 $4.9 \pm$ 1.0 vs $5.9 \pm 0.9, p<0.0019$ ) and functionality (mean HAQ $0.63 \pm 0.4$ vs $0.9 \pm 0.5, p<0.0019)$ were higher in pMDA than pRLDA respectively. Analysis within the pMDA group showed that patients in the plMDA subgroup were younger $(p=0.0005)$ with lower baseline disease activity (mean DAS28 $5.6 \pm 0.7$ vs $6.0 \pm 0.9, p<$ $0.0001)$ than phMDA patients. A representation of the DAS28 course for each patient in the pRLDA and pMDA groups is provided in Fig. 1 while Fig. 2 presents the 5-year disease activity course (average DAS28 in the 8 TT intervals) of groups pRLDA and pMDA and subgroups plMDA and phMDA, showing clear distinct disease activity trajectories.
pMDA group was associated with worse 5-year functionality than pRLDA

One of the main aims of this study was to investigate whether patients on pMDA have adverse long-term prognosis compared to patients on lower chronic inflammatory burden. The 5-year functionality (HAQ) trajectories (average DAS28 in the 8 TT intervals) of the pRLDA and pMDA groups are presented in Fig. 3, showing a clear distinct trajectory for each group. In multivariable mixed-effect regression analysis (Table 2), the pMDA group was associated with worse 5-year functionality trajectory than the pRLDA group $(+0.28$ higher HAQ trajectory in pMDA, $95 \% \mathrm{CI}+0.18$ to $+0.39, p<$ 0.0001 ). Analysis was adjusted for possible confounding effects on gender, age and disease duration. Interestingly, the 5-year functionality was also significantly worse in females than males $(+0.13$ higher HAQ trajectory in females, $95 \% \mathrm{CI}+0.04$ to $+0.23, p=0.008)$ and in older patients $(+0.009$ higher HAQ trajectory per 1 year, $95 \%$ $\mathrm{CI}+0.006$ to $+0.012, p<0.0001)$.

At 5 years, although both groups had improved functionality as compared to baseline (HAQ decrease from baseline: $-0.375 \mathrm{HAQ}, 95 \% \mathrm{CI}-0.448$ to $-0.303, p<$ 0.0001 ), pMDA group had worse functionality than pRLDA (HAQ $0.3 \pm 0.31$ vs $0.55 \pm 0.47, p<0.001$ ). Notably, most of the improvement occurred within the first 12 months of treatment (HAQ decrease from baseline in first 12 months: $-0.289,95 \% \mathrm{CI}-0.366$ to $-0.212, p<$ 0.0001 ), showing minimal additional improvement thereafter (12th up to 60th month).

\section{Subgroup phMDA was associated with worse 5-year functionality than pIMDA}

Clinical heterogeneity has been reported for the MDA group of patients on csDMARDs while limited data are available concerning bDMARDs. We assessed whether our cohort of RA patients on bDMARDs having persistent moderate disease activity represents a heterogeneous group. For this, we compared lower and higher pMDA subgroups, the pIMDA and phMDA, respectively. The 5 -year functionality trajectories (average HAQ in the 8 TT intervals) of the patients classified in plMDA and phMDA subgroups are presented in Fig. 3, revealing a clear distinct trajectory for each subgroup. In multivariable mixed-effect regression analysis (Table 3), the phMDA subgroup was associated with worse 5-year functionality trajectory than plMDA subgroup $(+0.26$ higher HAQ trajectory in pMDA, 95\% CI +0.17 to + $0.36, p<0.0001$ ). Analysis was adjusted for possible confounding effects on gender, age, and disease duration. The 5-year functionality was also significantly worse in females than males $(p=0.04)$ and in older patients $(p<$ $0.0001)$. At 5 years, the phMDA group had worse functionality status than plMDA (HAQ $0.41 \pm 0.38$ vs $0.66 \pm$ 
Table 1 Baseline characteristics of patient cohort and the different persistent disease activity groups

\begin{tabular}{|c|c|c|c|c|c|}
\hline \multirow[t]{2}{*}{ Variable name } & \multirow{2}{*}{$\begin{array}{l}\text { Cohort } \\
(n=385)\end{array}$} & \multicolumn{4}{|l|}{ Remission } \\
\hline & & $\begin{array}{l}\text { Low pRLDA }(n= \\
90)\end{array}$ & $\begin{array}{l}\text { Moderate pMDA ( } n= \\
\text { 295) }\end{array}$ & $\begin{array}{l}\text { Lower-moderate pIMDA } \\
(n=133)\end{array}$ & $\begin{array}{l}\text { Higher-moderate phMDA } \\
(n=162)\end{array}$ \\
\hline Females $^{a, b}$ & $\begin{array}{l}293 \\
(76 \%)\end{array}$ & $52(58 \%)$ & $241(82 \%)$ & $102(78 \%)$ & $139(86 \%)$ \\
\hline Age (years) $)^{a, b}$ & $\begin{array}{l}56.35 \pm \\
13\end{array}$ & $51.96 \pm 13$ & $57.69 \pm 12$ & $55.11 \pm 12.31$ & $59.81 \pm 11.59$ \\
\hline RA duration (years) & $9.2 \pm 8.6$ & $9.57 \pm 10$ & $9.1 \pm 8.12$ & $8.71 \pm 7.71$ & $9.43 \pm 8.45$ \\
\hline RA duration $<2$ years & $70(18 \%)$ & $22(24 \%)$ & $48(16 \%)$ & $20(15 \%)$ & $28(17 \%)$ \\
\hline Seropositive $^{*}(n=217)$ & $\begin{array}{l}100 \\
(46 \%)\end{array}$ & $15(47 \%)$ & $85(46 \%)$ & $37(51 \%)$ & $48(43 \%)$ \\
\hline $\operatorname{TJC}^{*} 8^{*}(n=316)^{\mathrm{a}, \mathrm{b}}$ & $\begin{array}{l}10.53 \pm \\
6.5\end{array}$ & $7.93 \pm 6.3$ & $11.14 \pm 6.4$ & $9.61 \pm 5.56$ & $12.39 \pm 6.74$ \\
\hline $\operatorname{SJC} 28^{*}(n=316)^{a, b}$ & $\begin{array}{l}8.99 \pm \\
6.24\end{array}$ & $6.17 \pm 6.1$ & $9.66 \pm 6.1$ & $8.14 \pm 5.55$ & $10.89 \pm 6.26$ \\
\hline $\mathrm{ESR}^{*} \mathrm{~mm} / \mathrm{s}(n=256)$ & $\begin{array}{l}38.62 \pm \\
24\end{array}$ & $35.37 \pm 23$ & $39.4 \pm 24$ & $37.86 \pm 23.42$ & $40.63 \pm 25.02$ \\
\hline $\mathrm{CRP}^{*} \mathrm{mg} / \mathrm{dl}(n=227)^{\mathrm{a}}$ & $2.57 \pm 6$ & $4.07 \pm 12$ & $2.22 \pm 3.15$ & $2.31 \pm 3.32$ & $2.15 \pm 3.02$ \\
\hline DAS28 (imputed) ${ }^{\mathrm{b}}$ & $\begin{array}{l}5.7 \pm \\
0.99\end{array}$ & $4.92 \pm 1$ & $5.94 \pm 0.86$ & $5.62 \pm 0.74$ & $6.02 \pm 0.86$ \\
\hline $\mathrm{CDAl}^{*}(n=296)^{\mathrm{b}}$ & $\begin{array}{l}33 \pm \\
12.19\end{array}$ & $26.06 \pm 12$ & $34.6 \pm 12$ & $30.86 \pm 10.05$ & $37.62 \pm 12.13$ \\
\hline $\operatorname{SDAl}^{*}(n=261)^{\mathrm{b}}$ & $\begin{array}{l}35.34 \pm \\
13\end{array}$ & $29.99 \pm 17$ & $36.64 \pm 12$ & $32.66 \pm 10.44$ & $39.63 \pm 12.18$ \\
\hline $\begin{array}{l}\text { Physician VAS G. }{ }^{*}(n= \\
298)^{\mathrm{a}, \mathrm{b}}\end{array}$ & $\begin{array}{l}69.5 \pm \\
14.8\end{array}$ & $62.92 \pm 16$ & $71.13 \pm 14$ & $67.57 \pm 15.12$ & $73.97 \pm 12.7$ \\
\hline Patient VAS G.* $(n=315)^{\mathrm{b}}$ & $\begin{array}{l}66.11 \pm \\
19\end{array}$ & $59.43 \pm 25$ & $67.69 \pm 17$ & $63.74 \pm 17.82$ & $70.93 \pm 15.54$ \\
\hline $\begin{array}{l}\text { Patient VAS Pain* }(n= \\
302)\end{array}$ & $\begin{array}{l}67.41 \pm \\
17\end{array}$ & $62.93 \pm 25$ & $68.5 \pm 17.2$ & $66.26 \pm 18.57$ & $70.38 \pm 15.87$ \\
\hline HAQ (imputed) ${ }^{a, b}$ & $\begin{array}{l}0.84 \pm \\
0.44\end{array}$ & $0.63 \pm 0.4$ & $0.9 \pm 0.5$ & $0.77 \pm 0.43$ & $1 \pm 0.45$ \\
\hline Euroqo* ${ }^{*}(n=82)$ & $\begin{array}{l}0.34 \pm \\
0.38\end{array}$ & $0.36 \pm 0.4$ & $0.34 \pm 0.39$ & $0.4 \pm 0.34$ & $0.26 \pm 0.43$ \\
\hline Previous csDMARDs & $\begin{array}{l}2.34 \pm \\
1.13\end{array}$ & $2.11 \pm 0.9$ & $2.41 \pm 1.18$ & $2.34 \pm 1.25$ & $2.46 \pm 1.11$ \\
\hline Ongoing csDMARDs & $\begin{array}{l}1.15 \pm \\
0.57\end{array}$ & $1.15 \pm 0.6$ & $1.15 \pm 0.6$ & $1.1 \pm 0.63$ & $1.19 \pm 0.53$ \\
\hline Monotherapy ${ }^{\mathrm{b}}$ & $32(8 \%)$ & $7(8 \%)$ & $25(9 \%)$ & $16(12 \%)$ & $9(6 \%)$ \\
\hline Methotrexate & $\begin{array}{l}279 \\
(73 \%)\end{array}$ & $62(69 \%)$ & $217(74 \%)$ & $97(73 \%)$ & $120(74 \%)$ \\
\hline Anti-TNF ${ }^{b}$ & $\begin{array}{l}350 \\
(90 \%)\end{array}$ & $88(98 \%)$ & $262(89 \%)$ & $124(93 \%)$ & $138(85 \%)$ \\
\hline Prednisolone & $\begin{array}{l}184 \\
(48 \%)\end{array}$ & 44 (49\%) & $140(48 \%)$ & $71(53 \%)$ & $69(43 \%)$ \\
\hline
\end{tabular}

Results presented as counts $n(\%)$ or mean $( \pm \mathrm{sd})$

*Missing data $>5 \%$

${ }^{a}$ Group pRLDA is significantly different from pMDA (Wilcoxon rank-sum test $p<0.05$ )

${ }^{\mathrm{b}}$ Group pIMDA is significantly different from phMDA (Wilcoxon rank-sum test $p<0.05$ )

TJC28 Tender Joint Count 28, SJC28 Swollen Joint Count 28, ESR erythrocyte sedimentation rate, CRP C reactive protein, DAS28 Disease Activity Score 28 ESR4, CDAI Clinical Disease Activity Index, SDAI Simplified Disease Activity Index, VAS visual analog scale 100, Physician VAS G. physician VAS global, Patient VAS G.

patient VAS global, HAQ Health Assessment Questionnaire, csDMARDs conventional synthetic disease-modifying antirheumatic drugs, Anti-TNF TNFa inhibitors 


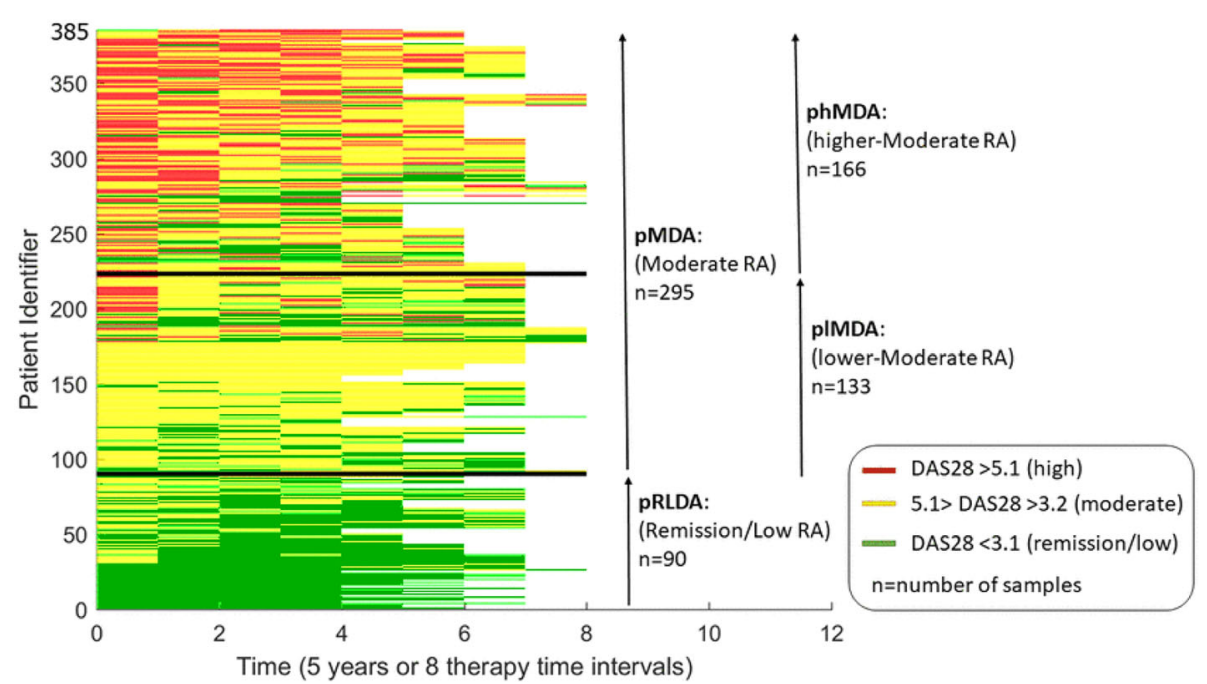

Fig. 1 DAS28 5-year disease activity course for each cohort patient (imputed data). RA, rheumatoid arthritis; DAS28, disease activity score with 28 joint counts; pRLDA, patients with persistent remission or low disease activity; pMDA, patients with persistent moderate disease activity; plMDA, patients with persistent lower-moderate disease activity; phMDA, patients with persistent higher-moderate disease activity

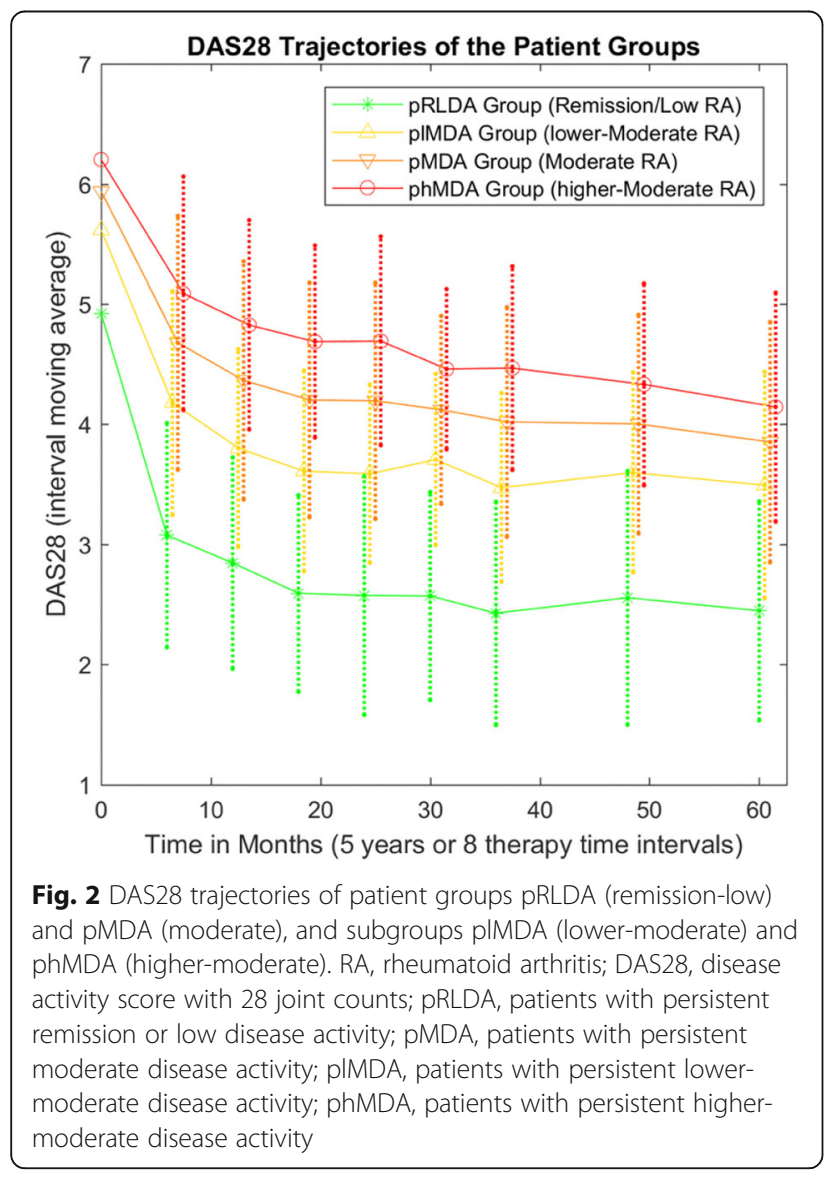

0.52 respectively, $p<0.001$ ), although both groups were associated with improved functionality as compared to baseline (HAQ decrease from baseline: - $0.38 \mathrm{HAQ}$, 95\% CI -0.463 to $-0.297, p<0.0001)$. The differentiation of 5 -year functionality between subgroups plMDA and phMDA indicates heterogeneity within the pMDA group.

\section{Patient (sub)groups are associated with different serious adverse events occurrence}

We also assessed SAEs occurrence during the course of the follow-up to estimate for any differences in the longterm outcome between distinct patients groups. The 5year cumulative SAEs trajectories of pRLDA and pMDA groups as well as of the pIMDA and phMDA subgroups are presented in Fig. 4, showing a clear distinct trajectory for each group and subgroup, respectively. At 5 years, pMDA group had higher occurrence of SAEs than pRLDA group $(0.2 \pm 0.48$ in pRLDA vs $0.5 \pm 0.96$ in pMDA, $p=0.006$ ). In addition, the phMDA subgroup had also higher occurrence of SAEs than the plMDA subgroup $(0.32 \pm 0.6$ in plMDA vs $0.64 \pm 1.16$ in phMDA, $p=0.038$ ). The differentiation between subgroups plMDA and phMDA in serious adverse events occurrence further supports the heterogeneity within the pMDA group.

\section{Early predictors for classification between pRLDA and pMDA groups}

In view of the aforementioned clinically meaningful differences between pRLDA and pMDA patient groups, we developed a predictive model for the early classification of patients using data from patients' early therapy 


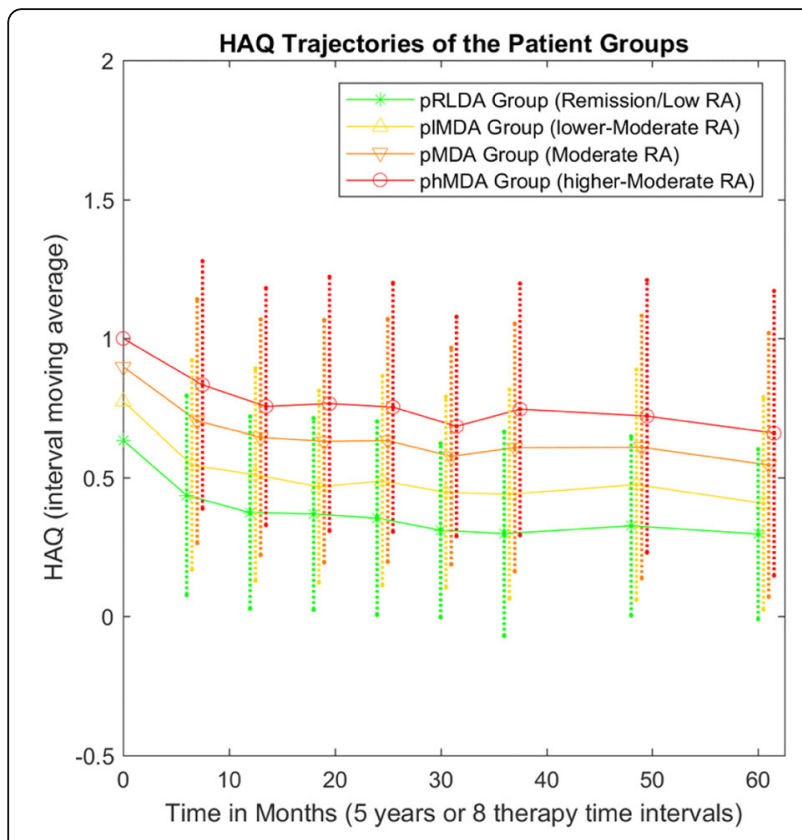

Fig. $3 \mathrm{HAQ}$ trajectories of patient groups pRLDA (remission-low) and pMDA (moderate) and subgroups pIMDA (lower-moderate) and phMDA (higher-moderate). RA, rheumatoid arthritis; HAQ,

functionality measured by Health Assessment Questionnaire; pRLDA, patients with persistent remission or low disease activity; pMDA, patients with persistent moderate disease activity; pIMDA, patients with persistent lower-moderate disease activity; phMDA, patients with persistent higher-moderate disease activity months (first semester of treatment). Multivariable logistic regression analysis (Table 4) was used adjusting for possible confounding effects of gender, age, disease duration, and previous csDMARDs (at baseline). Classification in the pRLDA group (compared to pMLDA group) was associated with male gender (female gender OR $0.38,95 \%$ CI 0.17 to $0.84, p=0.017$ ), lower baseline disease activity (DAS28 per unit: OR $0.45,95 \%$ CI 0.29 to $0.7, p<0.001$ ), lower first semester's average disease activity (DAS28-average per unit: OR $0.19,95 \%$ CI 0.12 to $0.29, p<0.001)$, and functionality improvement greater than 0.22 HAQ units in first semester's compared to baseline $(\triangle \mathrm{HAQ}<-0.22$ : OR $0.38,95 \%$ CI 0.16 to 0.91 , $p=0.029)$. Performance evaluation of the model in 10fold cross-validation process yielded $88.3 \%$ accuracy, $68 \%$ sensitivity, $95 \%$ specificity, and $91 \%$ area under the ROC curve.

\section{Discussion}

We characterized the long-term prognosis of RA patients with persistent moderate disease activity (MDA) under bDMARD treatment in the context of clinical practice. We found that a substantial proportion of patients improved disease activity status and function after treatment, yet a substantial proportion exhibited pMDA irrespective of treatment modifications. Most importantly, pMDA was associated with worse long-term (5year) outcomes (functional limitation and serious adverse events) than persistent lower inflammatory burden. Interestingly, the subgroup with plMDA had better long-term outcomes than those with phMDA.

Table 2 Multivariable mixed-effect regression associated group pMDA with worse 5-year functionality (HAQ) trajectory than pRLDA group

\begin{tabular}{llll}
\hline HAQ multivariable analysis $^{\dagger}$ & Coefficient $^{*}$ & $\mathbf{9 5 \%} \mathrm{Cl}$ & $\boldsymbol{p}$ value $^{*}$ \\
\hline Group pMDA (vs pRLDA) & +0.28 & +0.18 to +0.39 & $p<0.0001^{*}$ \\
Gender female (vs male) & +0.13 & +0.04 to +0.23 & $p=0.008^{*}$ \\
Age (per year) & +0.009 & +0.006 to +0.012 & $p<0.0001^{*}$ \\
Disease duration (per year) & +0.004 & -0.001 to +0.009 & $p=0.092$ \\
Time 3-9 months (vs baseline) & -0.203 & -0.279 to -0.126 & $p<0.0001^{*}$ \\
Time 9-15 months (vs baseline) & -0.289 & -0.366 to -0.212 & $p<0.0001^{*}$ \\
Time 15-21 months (vs baseline) & -0.288 & -0.364 to -0.213 & $p<0.0001^{*}$ \\
Time 21-27 months (vs baseline) & -0.285 & -0.362 to -0.208 & $p<0.0001^{*}$ \\
Time 27-33 months (vs baseline) & -0.347 & -0.432 to -0.261 & $p<0.0001^{*}$ \\
Time 33-42 months (vs baseline) & -0.326 & -0.399 to -0.254 & $p<0.0001^{*}$ \\
Time 42-54 months (vs baseline) & -0.323 & -0.396 to -0.251 & $p<0.0001^{*}$ \\
Time 54-60 months (vs baseline) & -0.375 & -0.448 to -0.303 & $p<0.0001^{*}$ \\
\hline
\end{tabular}

*Variable is associated significantly with patients' 5 -year functionality (HAQ) course ( $p<0.05$ significance threshold)

${ }^{\ddagger}$ Regression coefficient that represents increase $(+)$ or decrease $(-)$ in 5 -year functionality (HAQ) course associated with the variable (category membership for categorical variable or unit increase for continuous variable)

${ }^{\dagger}$ Efficiency of multivariable analysis: RMSE (Root mean square error) $=0.352, R^{2}(R$-squared) $=0.573$

$P R L D A$ persistent remission/low disease activity group; $P M D A$ persistent moderate disease activity group 
Table 3 Multivariable mixed-effect regression associated subgroup phMDA with worse 5-year functionality (HAQ) trajectory than pIMDA subgroup

\begin{tabular}{llll}
\hline HAQ multivariable analysis $^{\dagger}$ & Coefficient $^{*}$ & $\mathbf{9 5 \%}$ Cl & $p$ palue \\
\hline Group phMDA (vs plMDA) & +0.26 & +0.17 to +0.36 & $p<0.0001^{*}$ \\
Gender female (vs male) & +0.12 & +0.01 to +0.24 & $p=0.04^{*}$ \\
Age (per year) & +0.009 & +0.005 to +0.013 & $p<0.0001^{*}$ \\
Disease duration (per year) & +0.002 & -0.004 to +0.007 & $p=0.543$ \\
Time 3-9 months (vs baseline) & -0.200 & -0.287 to -0.114 & $p<0.0001^{*}$ \\
Time 9-15 months (vs baseline) & -0.285 & -0.373 to -0.196 & $p<0.0001^{*}$ \\
Time 15-21 months (vs baseline) & -0.291 & -0.378 to -0.204 & $p<0.0001^{*}$ \\
Time 21-27 months (vs baseline) & -0.279 & -0.369 to -0.190 & $p<0.0001^{*}$ \\
Time 27-33 months (vs baseline) & -0.342 & -0.444 to -0.240 & $p<0.0001^{*}$ \\
Time 33-42 months (vs baseline) & -0.308 & -0.392 to -0.225 & $p<0.0001^{*}$ \\
Time 42-54 months (vs baseline) & -0.313 & -0.396 to -0.231 & $p<0.0001^{*}$ \\
Time 54-60 months (vs baseline) & -0.380 & -0.463 to -0.297 & $p<0.0001^{*}$ \\
\hline
\end{tabular}

*Variable is associated significantly with patients' 5 -year functionality (HAQ) course $(p<0.05$ significance threshold)

${ }^{\ddagger}$ Regression coefficient that represents increase $(+)$ or decrease $(-)$ in 5 -year functionality (HAQ) course associated with the variable (category membership for categorical variable or unit increase for continuous variable)

${ }^{\dagger}$ Efficiency of multivariable analysis: RMSE (Root mean square error) $=0.366, R^{2}(R$-squared) $=0.538$

pIMDA persistent lower-moderate disease activity group; phMDA persistent higher-moderate disease activity group

An important finding of this study was that persistent MDA was linked to significantly worse functionality trajectory during 5 years of bDMARDs therapy, as compared to pRLDA (Fig. 3 and Table 2). Interestingly, residual disease activity was the major contributor to

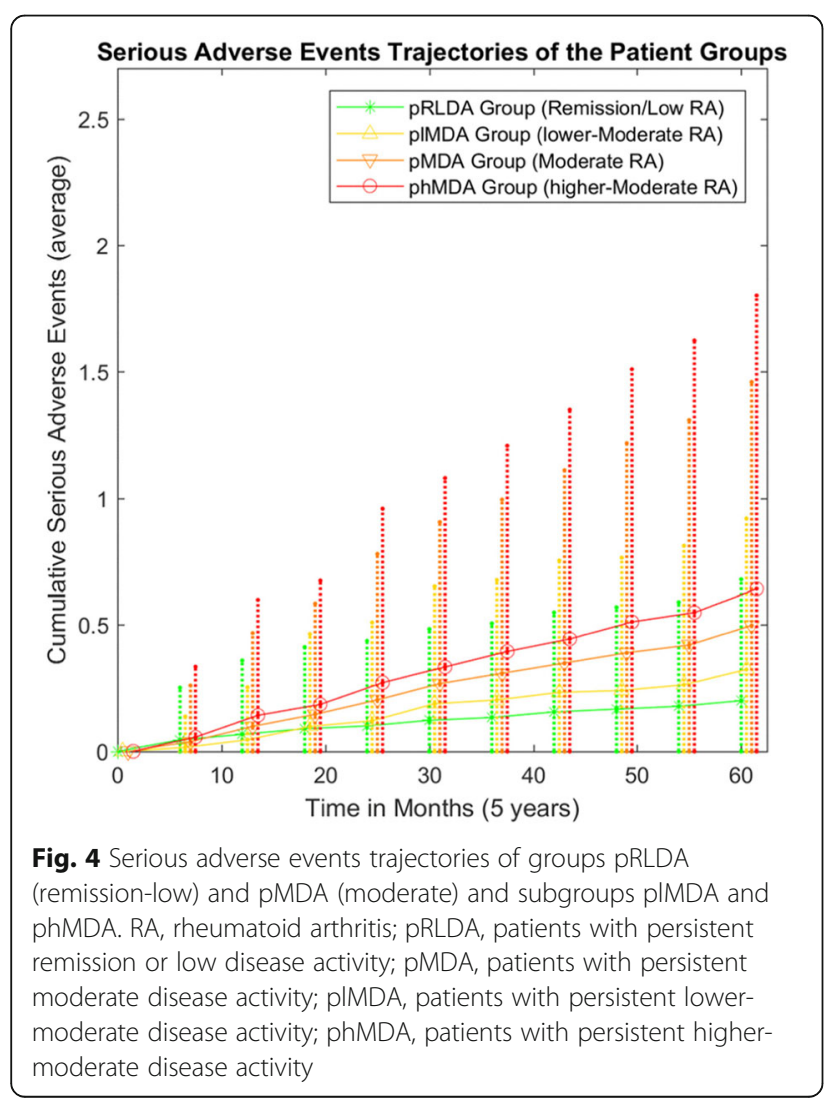

HAQ increase over time (Table 2). These data are comparable to those from early RA cohorts on csDMARDs assessing the cumulative effect of disease activity on RA-related outcomes [9, 10, 12]. Although one might argue that this finding is an expected one, we considered it clinically important and novel to focus for the first time on patients treated with bDMARDs, which might exert differential immunomodulatory effects as compared to csDMARDs. For example, it has been shown that both TNFi and tocilizumab may inhibit joint destruction effectively even when residual disease activity exists, which is not the case for methotrexate [13-15].

Another key finding of our study is that MDA patients comprise a heterogeneous group in terms of outcome. Patients with plMDA have significantly better 5-year functionality trajectory than those in phMDA (Fig. 3 and Table 3). This agrees with studies focusing on early-onset RA and csDMARDs treatments, showing that patients on the lower end of MDA have significantly better outcomes than those on the higher end $[10,16]$. Notwithstanding the fact that observational studies cannot provide direct support for management strategies, we consider our results to be of clinical importance and relevant to the T2T concept. Firstly, our data provide further support to the validity of T2T in clinical practice, since there was a clear superiority for all long-term outcomes in the pRLDA as compared to pMDA group. Moreover, the heterogeneity of outcomes in lower and higher MDA patients can assist T2T strategies to tailor treatments for these subgroups in order to improve outcomes. 
Table 4 Multivariable logistic regression analysis to predict classification in pRLDA compared to pMDA group

\begin{tabular}{|c|c|c|c|c|c|c|}
\hline \multirow{2}{*}{$\begin{array}{l}\text { pRLDA classification (vs } \\
\text { pMDA) }\end{array}$} & \multicolumn{3}{|c|}{ Univariable analysis } & \multicolumn{3}{|c|}{ Multivariable analysis $^{\dagger}$} \\
\hline & OR & $95 \% \mathrm{Cl}$ & $p$ value & OR & $95 \% \mathrm{Cl}$ & $p$ value \\
\hline Gender female (vs male) & 0.31 & 0.18 to 0.52 & $p<0.001^{*}$ & 0.38 & 0.17 to 0.84 & $p=0.017^{*}$ \\
\hline Age (per year) & 0.97 & 0.95 to 0.98 & $p<0.001^{*}$ & 0.98 & 0.96 to 1.02 & $p=0.26$ \\
\hline Disease duration (per year) & 1.01 & 0.98 to 1.03 & $p=0.65$ & 0.99 & 0.95 to 1.03 & $p=0.68$ \\
\hline Previous csDMARDs, count $<2$ & 1.56 & 0.91 to 2.67 & $p=0.11$ & 1.11 & 0.49 to 2.5 & $p=0.8$ \\
\hline Anti-TNF baseline & 5.54 & 1.3 to 23.57 & $p=0.02^{*}$ & 2.9 & 0.51 to 16.55 & $p=0.23$ \\
\hline DAS28 baseline (per unit) & 0.27 & 0.2 to 0.38 & $p<0.001^{*}$ & 0.45 & 0.29 to 0.7 & $p<0.001^{*}$ \\
\hline HAQ baseline (per unit) & 0.21 & 0.11 to 0.4 & $p<0.001^{*}$ & 0.53 & 0.2 to 1.38 & $p=0.19$ \\
\hline DAS28 1st semester (per unit) & 0.17 & 0.11 to 0.25 & $p<0.001^{*}$ & 0.19 & 0.12 to 0.29 & $p<0.001^{*}$ \\
\hline$\Delta \mathrm{HAQ}$ 1st semester $<-0.22$ & 0.65 & 0.37 to 1.15 & $p=0.14$ & 0.38 & 0.16 to 0.91 & $p=0.029^{*}$ \\
\hline
\end{tabular}

pRLDA persistent remission/low disease activity group, $p M D A$ persistent moderate disease activity group, $C D M A R D s$ conventional synthetic disease-modifying antirheumatic drugs, 1st semester therapy months 3-9, HAQ Health Assessment Questionnaire, $\triangle H A Q \mathrm{HAQ}$ difference 1 st semester's average from baseline, ACC accuracy, TPR sensitivity, TNR specificity, AUC area under receiver-operating-characteristic curve

${ }^{\dagger}$ Multivariable analysis efficiency (10-fold cross-validation): $A C C=88.3 \%, T P R=68 \%, T N R=95 \%, A U C=91 \%$

*Below significance threshold 0.05

An interesting finding was that patients on pMDA accumulated more SAEs compared to patients on pRLDA during 5 years of bDMARDs therapy (Fig. 4). Additionally, the analysis within pMDA showed that subgroup phMDA had more SAEs than plMDA at 5 years of therapy (Fig. 4). A major contributor in SAE is serious infections. The correlation between disease activity level and serious infections has been shown by several cohort studies [17-19]. Nevertheless, only our study and the analysis from the CORRONA registry by Accortt et al. are those analyzing "cumulative" disease activity levels, revealing the significant "dose effect" of inflammatory burden on the risk for serious infections [20]. Moreover, data from the Nijmegen early RA inception cohort have shown that time-averaged disease activity burden contributes to the risk of cardiovascular events in RA patients on different background therapies [21, 22]. These findings combined with the finding of higher functional decline of pMDA group underline the importance of cumulative residual disease activity as an important contributor in RA long-term prognosis.

Long-term prognosis of RA largely depends on the disease inflammatory burden and associated comorbidities. One of the limitations in the literature is that the majority of short- and even long-term studies evaluate RArelated inflammation cross-sectionally. However, values representative of longitudinal course of disease activity and its effect over time are considered to provide more valuable information. One such approach is the average disease activity from multiple years of treatment [10], while another is the area under the curve of DAS28 course (AUC) which was associated with both radiographic progression [23] and the risk for cardiovascular diseases (CVD) [21, 22]. In the present study, we applied another approach using the cumulative time percentage
(CTP) that DAS28 falls within a specified range during follow-up (CTP of DAS28-range), as indicative of longterm longitudinal disease activity course. We considered the AUC method not appropriate since it may not be able to distinguish a persistent moderate disease trajectory from one fluctuating equally between low and high disease activity levels which may exhibit approximately equivalent AUC values.

Studies analyzing persistent disease activity status from cohorts of biologics have focused on persistent remission [24-30]. Herein, we focused on persistent residual disease activity (pMDA), and we found that from patients in persistent moderate or lower inflammatory burden treated on bDMARDs, $23 \%$ were classified in a persistent low or remission status while $77 \%$ still exhibited substantial inflammatory burden (pMDA) after 5 years of therapy. Even though this seems as a rather high number, yet available data from registries have shown that only $8.2-21 \%$ of bDMARDs treated patients are classified as being in persistent remission [24-27, 29]. Of note, pMDA patients in our cohort differ from pRLDA even from baseline and could be divided further in two heterogeneous subgroups.

Early predictors for patient classification in pRLDA compared to pMDA group identified in multivariable predictive modeling were male gender, lower baseline, and lower first semester disease activity (DAS28) and functionality improvement in first semester compared to baseline $(\triangle \mathrm{HAQ}<-0.22)$ (Table 4). Comparable to our data, a meta-analysis of six studies for factors associated to sustained remission in patients treated with TNF inhibitors showed that greater baseline disease activity, age, disease duration, baseline functional impairment, and female sex were associated with reduced likelihood of achieving sustained remission [30]. Nevertheless, 
prediction of response at individual level is not yet clinically available. Future studies should enrich the predictive models with additional biological parameters aiming to further increase the predictive performance of current tools.

One of the limitations of this study is patients' missing follow-up data. In order to address missing data and also maintain data quality, we excluded patients with large percentage of missing DAS28-data (>50\%) and imputed missing DAS28-data in the rest of the patients. Of note, the imputed-dataset (385 patients) compared to the non-imputed (292 patients) included additionally 93 (24\%) patients, and results were similar in both datasets (groups pRLDA and pMDA differentiated in functionality trajectory and SAEs, data not shown).

Another limitation of this study can be considered the merging of remission and low disease activity groups. The pRLDA group included 52 patients in persistent remission, 20 in persistent low disease activity, and 18 with fluctuations between low and remission disease activity. Future studies in larger datasets could focus in persistent strictly low $(2.6 \leq \mathrm{DAS} 28 \leq 3.2)$ and persistent moderate $(3.2<$ DAS28 $\leq 5.1)$ disease activity comparison.

In order to evaluate the robustness of the methodological approach, we performed sensitivity analysis in shorter (3-year) therapy duration with similar results (groups pRLDA and pMDA differentiated in functionality trajectory). Furthermore, analysis on groups' differences regarding patients' inclusion-year (year $\leq 2007$, 2007 < year $\leq 2010$, year $>2010$ ) did not yield significant variation between the groups.

Conclusions Our analysis revealed that a considerable proportion of RA patients on bDMARDs in clinical practice, although improve disease activity status, still manifests persistent moderate disease activity. This state was associated with adverse 5-year outcomes and was also found to present internal heterogeneity, while predictors were analyzed to assist for early patient classification. These findings further support the value of T2T strategy in order to improve long-term outcome and highlight the need for further targeted studies on persistent MDA state and its heterogeneous subgroups.

\footnotetext{
Abbreviations

RA: Rheumatoid arthritis; MDA: Moderate disease activity; pMDA: Persistent moderate disease activity; pRLDA: Persistent remission or low disease activity; pIMDA: Persistent lower-moderate disease activity; phMDA: Persistent highermoderate disease activity; HAQ: Health Assessment Questionnaire for Functionality Assessment; SAEs: Serious adverse events; bDMARDs: Biologic disease-modifying antirheumatic drugs; csDMARDs: Conventional synthetic disease-modifying antirheumatic drugs; HeRBT: Hellenic Registry of Biologic Therapies; DAS28: Disease activity score of 28 joint counts; TNFis: TNFa inhibitors; TT: Clinically relevant therapy time intervals; CTP: Cumulative time percentage; $\triangle \mathrm{HAQ}$ : HAQ difference; ACC: Accuracy; TPR: Sensitivity or true positive rate; TNR: Specificity or true negative pate; AUC: Area under the receiver-operating-characteristic curve; T2T: Treat to target
}

\section{Acknowledgements}

The authors acknowledge the collaboration of all consultant rheumatologists and their specialist nurses for the acquisition, maintenance, and provision of the data. Specifically, the authors thank the following collaborators: Argyro Repa (MD), Nestor Avgoustidis (MD), Nikolaos Kougkas (MD), loannis Papalopoulos (MD), Styliani Polia (RN), and Eleni Kampouraki (RN) from the Rheumatology and Clinical Immunology Clinic, University General Hospital of Heraklion (PAGNI), Heraklion, Crete, Greece.

\section{Authors' contributions}

$I G$, IF, and PS authors were involved in the design and statistical analysis of the study as well as manuscript drafting and approval of the final version while the rest of the authors contributed equally in consulting, manuscript proofreading, and approval.

\section{Funding}

This work was supported in 2018 by the Hellenic Foundation for Research and Innovation (HFRI) and the General Secretariat for Research and Technology (GSRT), under the HFRI PhD Fellowship grant (no. 1722), under the HFRI PhD Fellowship grant [grant number 1722].

\section{Availability of data and materials}

The data and analytic methods that support the findings of this study are available to qualified investigators by request to the corresponding author.

\section{Ethics approval and consent to participate}

Ethical approval was obtained from the Institutional Review-Board of the University Hospital of Heraklion, Crete (decision number 1476/20-03-2012). Participants provided informed consents.

Consent for publication

Not applicable.

\section{Competing interests}

The authors declare that they have no competing interests.

\section{Author details}

${ }^{1}$ Rheumatology and Clinical Immunology Department, School of Medicine at University of Crete, Voutes, 71110 Heraklion, Greece. ${ }^{2}$ Institute of Computer Science at Foundation for Research and Technology Hellas, Heraklion, Greece. ${ }^{3}$ Informatics Engineering Department, Technological Educational Institute of Crete, Heraklion, Greece. ${ }^{4}$ Rheumatology Department, Sismanoglio Hospital, Athens, Greece. ${ }^{5}$ Nutrition and Dietetics Department, Harokopio University of Athens, Athens, Greece. ${ }^{6}$ Rheumatology Department, School of Medicine at University of loannina, Ioannina, Greece. ${ }^{7} 4$ th Internal Medicine Department, School of Medicine at National and Kapodistrian University of Athens, Athens, Greece.

Received: 8 July 2020 Accepted: 7 September 2020

Published online: 29 September 2020

\section{References}

1. Aletaha D, Funovits J, Smolen JS. The importance of reporting disease activity states in rheumatoid arthritis clinical trials. Arthritis Rheum. 2008; 58(9):2622-31.

2. Smolen JS, Breedveld FC, Burmester GR, Bykerk V, Dougados M, Emery P, et al. Treating rheumatoid arthritis to target: 2014 update of the recommendations of an international task force. Ann Rheum Dis. 2016;75(1): $3-15$

3. Smolen JS, Landewe R, Bijlsma J, Burmester G, Chatzidionysiou K, Dougados $M$, et al. EULAR recommendations for the management of rheumatoid arthritis with synthetic and biological disease-modifying antirheumatic drugs: 2016 update. Ann Rheum Dis. 2017;76(6):960-77.

4. Hetland M, Christensen I, Tarp U, Dreyer L, Hansen A, Hansen I, et al. Direct comparison of treatment responses, remission rates, and drug adherence in patients with rheumatoid arthritis treated with adalimumab, etanercept, or infliximab: results from eight years of surveillance of clinical practice in the nationwide Danish DANBIO registry. Arthritis Rheum. 2010;62(1):22-54.

5. Greenberg JD, Reed G, Decktor D, Harrold L, Furst D, Gibofsky A, et al. A comparative effectiveness study of adalimumab, etanercept and infliximab 
in biologically naive and switched rheumatoid arthritis patients: results from the US CORRONA registry. Ann Rheum Dis. 2012;71(7):1134-42.

6. Flouri I, Markatseli TE, Voulgari PV, Boki KA, Papadopoulos I, Settas L, et al. Comparative effectiveness and survival of infliximab, adalimumab, and etanercept for rheumatoid arthritis patients in the Hellenic Registry of Biologics: low rates of remission and 5-year drug survival. Semin Arthritis Rheum. 2014;43(4):447-57.

7. Gabay C, Riek M, Scherer A, Finckh A. Effectiveness of biologic DMARDs in monotherapy versus in combination with synthetic DMARDs in rheumatoid arthritis: data from the Swiss Clinical Quality Management Registry. Rheumatology (Oxford). 2015;54(9):1664-72.

8. Forsblad-d'Elia H, Bengtsson K, Kristensen LE, Jacobsson LT. Drug adherence, response and predictors thereof for tocilizumab in patients with rheumatoid arthritis: results from the Swedish biologics register. Rheumatology (Oxford). 2015;54(7):1186-93.

9. Combe B, Logeart I, Belkacemi MC, Dadoun S, Schaeverbeke T, Daures JP, et al. Comparison of the long-term outcome for patients with rheumatoid arthritis with persistent moderate disease activity or disease remission during the first year after diagnosis: data from the ESPOIR cohort. Ann Rheum Dis. 2015;74(4):724-9.

10. Nikiphorou E, Norton S, Young A, Carpenter L, Dixey J, Walsh DA, et al. Association between rheumatoid arthritis disease activity, progression of functional limitation and long-term risk of orthopaedic surgery: combined analysis of two prospective cohorts supports EULAR treat to target DAS thresholds. Ann Rheum Dis. 2016;75(12):2080-6.

11. Flouri ID, Markatseli TE, Boki KA, Papadopoulos I, Skopouli FN, Voulgari PV, et al. Comparative analysis and predictors of 10-year tumor necrosis factor inhibitors drug survival in patients with spondyloarthritis: first-year response predicts longterm drug persistence. J Rheumatol. 2018;45(6):785-94.

12. Cook MJ, Diffin J, Scire CA, Lunt M, MacGregor AJ, Symmons DP, et al, Predictors and outcomes of sustained, intermittent or never achieving remission in patients with recent onset inflammatory polyarthritis: results from the Norfolk Arthritis Register. Rheumatology. 2016;55(9):1601-9.

13. Landewe $R$, van der Heijde $D$, Klareskog $L$, van Vollenhoven $R$, Fatenejad $S$. Disconnect between inflammation and joint destruction after treatment with etanercept plus methotrexate: results from the trial of etanercept and methotrexate with radiographic and patient outcomes. Arthritis Rheum. 2006;54(10):3119-25.

14. Smolen JS, Han C, Bala M, Maini RN, Kalden JR, van der Heijde D, et al. Evidence of radiographic benefit of treatment with infliximab plus methotrexate in rheumatoid arthritis patients who had no clinical improvement: a detailed subanalysis of data from the anti-tumor necrosis factor trial in rheumatoid arthritis with concomitant therapy study. Arthritis Rheum. 2005;52(4):1020-30.

15. Smolen JS, Avila JC, Aletaha D. Tocilizumab inhibits progression of joint damage in rheumatoid arthritis irrespective of its anti-inflammatory effects: disassociation of the link between inflammation and destruction. Ann Rheum Dis. 2012;71(5):687-93.

16. Kiely $P$, Walsh $D$, Williams $R$, Young A, Early Rheumatoid Arthritis N. Outcome in rheumatoid arthritis patients with continued conventional therapy for moderate disease activity--the early RA network (ERAN). Rheumatology (Oxford). 2011;50(5):926-31.

17. Mehta B, Pedro S, Ozen G, Kalil A, Wolfe F, Mikuls T, et al. Serious infection risk in rheumatoid arthritis compared with non-inflammatory rheumatic and musculoskeletal diseases: a US national cohort study. RMD open. 2019;5(1):e000935.

18. Au K, Reed G, Curtis JR, Kremer JM, Greenberg JD, Strand V, et al. High disease activity is associated with an increased risk of infection in patients with rheumatoid arthritis. Ann Rheum Dis. 2011;70(5):785-91.

19. Weaver A, Troum O, Hooper M, Koenig AS, Chaudhari S, Feng J, et al. Rheumatoid arthritis disease activity and disability affect the risk of serious infection events in RADIUS 1. J Rheumatol. 2013;40(8):1275-81.

20. Accortt NA, Lesperance T, Liu M, Rebello S, Trivedi M, Li Y, et al. Impact of sustained remission on the risk of serious infection in patients with rheumatoid arthritis. Arthritis Care Research. 2018;70(5):679-84.

21. Arts EE, Fransen J, den Broeder AA, Popa CD, van Riel PL. The effect of disease duration and disease activity on the risk of cardiovascular disease in rheumatoid arthritis patients. Ann Rheum Dis. 2015;74(6):998-1003.

22. Arts EE, Fransen J, Den Broeder AA, van Riel PL, Popa CD. Low disease activity (DAS28 3.2 ) reduces the risk of first cardiovascular event in rheumatoid arthritis: a time-dependent cox regression analysis in a large cohort study. Ann Rheum Dis. 2017;76(10):1693-9.
23. Tsuji H, Yano K, Furu M, Yamakawa N, Ikari K, Hashimoto M, et al. Timeaveraged disease activity fits better joint destruction in rheumatoid arthritis. Sci Rep. 2017;7(1):5856.

24. Broca O, Millasseau E, Albert C, Grisot C, Flory P, Roux CH, et al. Effect of discontinuing TNFalpha antagonist therapy in patients with remission of rheumatoid arthritis. Joint Bone Spine. 2009;76(4):350-5.

25. Furst DE, Pangan AL, Harrold LR, Chang H, Reed G, Kremer JM, et al. Greater likelihood of remission in rheumatoid arthritis patients treated earlier in the disease course: results from the Consortium of Rheumatology Researchers of North America registry. Arthritis Care Res (Hoboken). 2011;63(6):856-64.

26. Einarsson JT, Geborek P, Saxne T, Kapetanovic MC. Sustained remission in tumor necrosis factor inhibitor-treated patients with rheumatoid arthritis: a population-based cohort study. J Rheumatol. 2015;42(5):741-8.

27. Balogh E, Madruga Dias J, Orr C, Mullan R, Harty L, FitzGerald O, et al. Comparison of remission criteria in a tumour necrosis factor inhibitor treated rheumatoid arthritis longitudinal cohort: patient global health is a confounder. Arthritis Res Ther. 2013;15(6):R221.

28. Tanaka Y, Hirata S, Kubo S, Fukuyo S, Hanami K, Sawamukai N, et al. Discontinuation of adalimumab after achieving remission in patients with established rheumatoid arthritis: 1-year outcome of the HONOR study. Ann Rheum Dis. 2015;74(2):389-95.

29. Barnabe C, Homik J, Barr SG, Martin L, Maksymowych WP. The effect of different remission definitions on identification of predictors of both point and sustained remission in rheumatoid arthritis treated with anti-TNF therapy. J Rheumatol. 2014;41 (8):1607-13.

30. Hamann P. Holland R, Hyrich K, Pauling JD, Shaddick G, Nightingale A, et al. Factors associated with sustained remission in rheumatoid arthritis in patients treated with anti-tumor necrosis factor. Arthritis Care Res (Hoboken). 2017;69(6):783-93.

\section{Publisher's Note}

Springer Nature remains neutral with regard to jurisdictional claims in published maps and institutional affiliations.
Ready to submit your research? Choose BMC and benefit from:
- fast, convenient online submission
- thorough peer review by experienced researchers in your field
- rapid publication on acceptance
- support for research data, including large and complex data types
- gold Open Access which fosters wider collaboration and increased citations
- maximum visibility for your research: over $100 \mathrm{M}$ website views per year
At BMC, research is always in progress.
Learn more biomedcentral.com/submissions 